\title{
Association Between High Immune Activity and Worse Prognosis in Uveal Melanoma and Low- Grade Glioma in TCGA Transcriptomic Data
}

Hitoshi Matsuo

The University of Tokyo

Takashi Kamatani

The University of Tokyo

Yu Hamba

The University of Tokyo

Keith A. Boroevich

RIKEN Center for Integrative Medical Sciences

Tatsuhiko Tsunoda ( $\nabla$ tsunoda@bs.s.u-tokyo.ac.jp )

The University of Tokyo

\section{Research Article}

Keywords: endothelial mesenchymal transition, glioma, inflammation, macrophages, uveal melanoma

Posted Date: August 23rd, 2021

DOI: https://doi.org/10.21203/rs.3.rs-744639/v1

License: (9) This work is licensed under a Creative Commons Attribution 4.0 International License. Read Full License 


\section{Abstract}

Background: Immune status in the tumor microenvironment is an important determinant of cancer progression and patient prognosis. Although a higher immune activity is often associated with a better prognosis, this trend is not absolute and differs across cancer types. We aimed to give insights into why some cancers do not show better survival despite higher immunity by assessing the relationship between different biological factors, including cytotoxicity, and patient prognosis in various cancer types using RNA-seq data collected by The Cancer Genome Atlas.

Results: Results showed that a higher immune activity was associated with worse prognosis in patients with uveal melanoma and low-grade glioma, which are cancers of immune-privileged sites. In these cancers, epithelial or endothelial mesenchymal transition and inflammatory state as well as immune activation had notably negative correlation with patient prognosis. Further analysis using additional single-cell data of uveal melanoma and glioma revealed that epithelial or endothelial mesenchymal transition was mainly induced in retinal pigment cells or endothelial cells that comprise the blood-retinal and blood-brain barriers, which are unique structures of the eye and central nervous system, respectively. Inflammation was mainly promoted by macrophages, and their infiltration increased significantly in response to immune activation. Furthermore, we found the expression of CCL5 was strongly correlated with immune activity and associated with bad prognosis, particularly in these cancers, suggesting that CCL5 is a potential molecular target for therapeutics.

Conclusions: Epithelial or endothelial mesenchymal transition is associated with a bad prognosis. This suggests that inflammation loosens the structures of the blood barrier and causes further infiltration of immune cells, which may result in a feedback loop of additional inflammatory effects leading to runaway conditions.

\section{Background}

Immune status in the tumor microenvironment (TME) is important in determining cancer progression and patient prognosis. The effect of immune cell infiltration or immune activity level in the TME has received significant attention in the past decade. Cytotoxic T cells (CTLs) play an essential role in cancer elimination as they induce apoptosis in recognized cancer cells by releasing cytokines such as perforin and granzyme. Moreover, in tumors highly infiltrated with CTLs, cancer elimination occurs actively. In some tumors, CTL activity is inhibited because there is minimal CTL infiltration or cancer cells express checkpoint molecules and acquire systems to prevent attack by CTLs [1,2]. Generally, tumors with a high cytotoxicity are correlated with a better patient prognosis [3], and therapies that activate tumoral immunity or enhance cytotoxicity have been developed $[4,5,6,7]$.

However, some reports have shown that a high immune activity or immune cell infiltration is associated with a worse prognosis in certain cancer types $[7,8]$. The abovementioned trend in cancer immunology is not common, and it may be affected by cancer type-specific factors. We focused on uveal melanoma 
(UVM) and glioma, which are cancers of the eye and central nervous system (CNS), respectively. These sites have a common anatomical structure, referred to as the blood-retinal barrier (BRB) in the eye and blood-brain barrier (BBB) in the CNS. The entry of immune cells into the tissues is regulated. Hence, both organs are referred to as immune-privileged sites, and have the immune systems different from other organs. We hypothesized that the prognostic impact of varying immune statuses could differ among them. Thus, the current study aimed to investigate how cytotoxicity and biological states and processes affect prognosis in UVM and glioma using transcriptomic data from The Cancer Genome Atlas. Moreover, to assess poor prognostic factors specific to these cancer types, this correlation was compared across other cancer types. Subsequently, an analysis with additional single-cell data determined from which cells these factors originate, and the mechanisms associated with poor prognosis and histological features were identified.

\section{Results}

\section{Association between high immune activity and poor prognosis in patients with UVM and LGG}

To evaluate immune activity status in the TME, we assessed the CTL levels in all TCGA samples and performed a survival analysis between the high and low CTL groups for each cancer type (Fig. S1a, S1b and S1c). In most cancers, including SKCM, the prognosis was significantly better in the high CTL group than in the low CTL group. However, in patients with UVM and low-grade glioma (LGG), the low CTL group had a significantly better prognosis than the high CTL group (Fig. 1a and S1C). Moreover, the prognosis did not differ between high and low CTL groups in patients with glioblastoma (GBM), which is a highgrade glioma. The CTL level was higher in patients with GBM than in those with LGG (Fig. S1a). To validate these findings, we performed the same analysis for gliomas in the CGGA dataset and found that high CTL level was associated with a worse prognosis in LGG. Moreover, the immune activity of GBM was higher than that of LGG (Fig. S1b). The high CTL group had higher PD-L1 (CD274) expression than the low CTL group, which was common in all cancer types (Fig. S1d).

Next, we assessed well-known biological states and processes in the Hallmark gene sets registered in MSigDB, by calculating the activities of these states and processes in all TCGA samples via SSGSEA. A dimensional compression analysis using tSNE showed clustering according to cancer type and organs with similar functions were plotted adjacent to each other (Fig. 1b). Interestingly, UVM and SKCM were almost in the same position (Fig. 1b). Melanocyte-derived cancers had similar biological states and processes compared with other types of cancers. The relationships between immune activity and prognosis were completely reversed between UVM and SKCM. Similarly, LGG and GBM clustered tightly with each other. Therefore, in the succeeding analysis, we focused on the differences between UVM and SKCM as well as LGG and GBM to assess the prognostic factors of UVM and LGG.

\section{Hazard ratios of inflammation and EMT in UVM and LGG}


To examine which biological states and processes in SSGSEA that were strongly associated with worse prognosis in UVM and LGG, we calculated their hazard ratios (HRs). Results showed that epithelial mesenchymal transition (EMT) had the highest HR for worse prognosis in LGG (Fig. 2a). On the other hand, the HR of EMT in GBM was close to 1 (Fig. 2a; Table S1). In LGG, the activity score of EMT was positively correlated with the CTL level and was significantly enhanced in the high CTL group compared with the low CTL group (Fig. 2b and S2c; Table S2). Moreover, these results were confirmed via an analysis using the CGGA dataset (Fig. S2a and S2b). To investigate the cause of the enhanced EMT and immune activation, we focused on the upstream signaling pathways associated with the former. In gliomas, EMT is promoted by the TGF-beta, Wnt-beta catenin, Notch, and Hedgehog signaling pathways $[9,10,11]$. The TGF-beta pathway alone was found to be minimally activated in the high CTL group, as assessed using the TCGA dataset (Fig. 2b). However, this result could not be validated using the CGGA dataset (Fig. S2b). The activity of the Notch and Hedgehog signaling pathways was not correlated with the differences in immune activation status, and there was greater Wnt-beta activation in the low CTL group than in the high CTL group (Fig. 2b, S2b, S2c and S2d). By contrast, inflammation and hypoxia are also known to enhance EMT in several brain diseases $[9,11]$, and these scores were significantly increased in the high CTL group in our results (Fig. 2b). The activities of the inflammatory signaling pathways, such as the IL2-STAT5, IL6-STAT3, interferon-alpha (INF-a), interferon-gamma (INF- $y$ ), and tumor necrosis factor alpha (TNF-a), were positively correlated with the CTL level and were significantly more activated in the high CTL group compared to the low CTL group (Fig. 2b, S2b, S2c and S2d; Table S2). Furthermore, the HRs of these pathways were higher in LGG than in GBM (Fig. S2e).

Next, we compared the HRs of each biological state/process for worse prognosis between UVM and SKCM. The IL6-JAK-STAT3, IL2-STAT5, INF-a/ $Y$, and TNF-a pathways had a significantly higher and lower HRs in UVM and SKCM, respectively (Fig. 2a; Table S3). The activities of these signaling pathways were positively correlated with CTL levels and were significantly activated in the high CTL group (Fig. $2 \mathrm{~b}$ and S3a; Table S4). The HR of the EMT in LGG was significantly higher in UVM, and it was close to 1 in SKCM (Fig. 2a). In some eye diseases, EMT is enhanced after inflammation or the activation of the TGF-beta pathway [12]. Our results show that inflammation was enhanced, as described above, and the TGF-beta pathway was significantly activated in the high CTL group (Fig. 2b, S3a). Among all of the cancer types in TCGA, UVM and LGG had the two highest HRs of EMT, inflammatory response and hypoxia for worse prognosis (Fig. 2c and S3b).

\section{Types of cells causing inflammatory effects and EMT in UVM and LGG}

As presented in the previous section, inflammatory effects and EMT were found to be strongly correlated with worse prognosis in UVM and LGG. To determine which cells are responsible for the inflammatory effects and EMT, we used single-cell RNA-seq data from GEO (GSE139829 and GSE138794) [13, 14]. We calculated the activity score of IL6-STAT3, IL2-STAT5, INF- $a$ / $y$, TNF- $a$, and inflammatory states via sSGSEA using the single-cell data of eight primary UVM samples. The Umap analysis revealed several cell 
clusters with high inflammatory effects (Fig. 3a and S4). In these clusters, CD68 and CD163 are expressed, both of which are known markers of macrophages (Fig. 3a, S4a and b). Moreover, cell populations with EMT had a high expression of endothelial and retinal pigmented epithelial cell markers (Fig. 3a, S4a and S4b). From the single-cell data of nine glioma samples, we identified inflammatory cell populations that were positive for CD68 (Fig. 3b, S4c and d). In contrast to UVM, CD163 positive cells showed only a slight inflammatory response in glioma (Fig. 3b, S4c and d). The cell populations in which EMT was activated were mainly cells expressing endothelial cell markers, similar to what was seen in UVM (Fig. 3b, S4c and d). Because CD163 indicates M2-like macrophages, the inflammatory effect, which was associated with worse prognosis in UVM and LGG, is mainly observed in macrophage populations that differ in phenotype from each other: M2-like macrophages in UVM and M0/M1-like macrophages in LGG.

Next, to validate whether tumors with a higher immune activity have an increased abundance of macrophages, we evaluated the immune status of the tumors using CIBERSORT. The absolute abundance scores of M1 and M2 macrophages in UVM were positively correlated with the CTL level. The infiltration of these macrophages was advanced in the state of high immune activity (Fig. 3c and S5a). The ratios of each immune cell showed that M2 macrophages were the most predominant cells in UVM (Fig. S5c). The percentages of macrophages and monocytes in the tumor were compared between UVM and SKCM. Results showed that M2 macrophages were more abundant in UVM than in SKCM (Fig. 3d).

In LGG, the proportion of M0, M1, and M2 macrophages was significantly increased in tumors with higher CTL levels, and a similar correlation was observed in the xCell scores (Fig. 3e and S5b). Furthermore, a survival analysis was performed by dividing the absolute scores of M1 and M2 macrophages by the median. Results showed that a worse prognosis was significantly associated with a high macrophage invasion (Fig. S5c). Because the M0 macrophage score was 0 in more than half of the samples, survival analysis could not be performed for $\mathrm{M} 0$. In addition, we compared the percentage of macrophages (combined monocyte and M0, M1, and M2 macrophages) in all cancer types. In LGG and GBM, the macrophages were significantly abundant, and glioma was a macrophage-dominant cancer (Fig. S5f). By comparing the percentages of macrophages in LGG and GBM, the fraction of $\mathrm{M} 0$ and $\mathrm{M} 1$ macrophages increased in GBM (Fig. 3f). Results showed that increased infiltration of macrophages was associated well with immune activation.

\section{High correlation between the expression of chemokine CCL5 and CTL level}

We investigated potential molecules that can improve the function of BRB and BBB and inhibit the infiltration of immune cells, such as macrophages. Here we focused on chemokines because they are a class of cytokines involved in the development of inflammation by promoting the migration of leukocytes and other immune cells, and they have recently attracted attention as targets for cancer therapeutics. We calculated the HR of each chemokine for worse prognosis based on expression levels. Results showed 
that CCL5 had the highest HR in UVM (Fig. 4a; Table S5). CCL5 is known to promote BBB disruption, and the HR of CCL 5 was significantly high in LGG, whereas it was low in SKCM and close to 1 in GBM. The expression of CCL5 was most positively correlated with CTL level in LGG, and a high correlation was also observed in UVM (Fig. 4b; Table S6). CXCL11, which is a ligand of CXCR3, had the highest HR in LGG. CXCR3 has ligands including CXCL9, CXCL10, and CXCL11. These chemokines had high HRs in both UVM and LGG, and they were also strongly correlated with CTL levels (Tables S5, S6 and S7).

\section{Discussion}

Contrary to the general trend of tumor immunity, the prognosis was significantly worse in the high CTL group than in the low CTL group in UVM and LGG. On the other hand, in SKCM, to which checkpoint inhibitor was first applied, the prognosis was significantly better in the high CTL group. A tSNE plot generated based on the activity scores of well-defined biological states and processes as determined via SSGSEA showed that UVM and SKCM, both of which are derived from melanocytes, resided in almost the same cluster, indicating that they share common biological properties. Therefore, high immune activity being associated with a poor prognosis in UVM is likely due to the tissue-specific histological nature of the eye rather than the molecular endogenous characteristics of each cell within the tumor. The BRB and BBB are the common structures of the eye and CNS; these are physical barriers created by tight junctions around vascular endothelial cells that line the space between capillaries and tissues and various cells surrounding them (such as optic nerve cells in the eye or pericytes and astrocytes in the CNS). The outer BRB is comprised of pigmented retinal epithelial cells, and the inner BRB of capillary endothelial cells [15]. These structures prevent immune cell infiltration $[16,17,18]$, and may contribute to the low CTL level observed in UVM and LGG compared to other cancer types, which suggests that this histological feature is a factor of the poor prognosis in cases of high cytotoxicity.

Inflammatory effects and EMT were strong poor prognostic indicators of UVM and LGG. Furthermore, most inflammatory effects were seen in macrophage populations, and EMT was observed in epithelial and endothelial cell populations. Endothelial mesenchymal transition (EndoMT) is almost the same conversion mechanism as EMT induced in endothelial cells, suggesting that EMT from ssGSEA found in endothelial cells is a proxy for EndoMT. In some eye and brain diseases, the epithelial and endothelial cells comprising the BRB and BBB are known to cause EMT and EndoMT, respectively $[9,12]$. Therefore, the same mechanism can occur in UVM and LGG. In particular, EMT was associated the most with a worse prognosis in LGG. In the brain, the TGF-beta, Wnt-beta catenin, NOTCH, and Hedgehog signal pathways activate EMT and EndoMT $[9,10,11]$, but there were not sufficient results to show that these pathways were activated in the high CTL group. On the other hand, inflammatory state and hypoxia, which were significantly enhanced in the High-CTL group, are also known to promote EMT/EndoMT in brain. These states increase the permeability of the BBB and makes endothelial cells more prone to exhibit EndoMT and also loosen the tight junctions of the BBB $[9,11]$. The current study suggests that with immune activation, macrophage infiltration increased, and the inflammatory effects also became 
stronger. Furthermore, compared with other cancer types, inflammation response and hypoxia were strongly associated with worse prognosis in LGG, indicating that inflammation and hypoxia may be strong contributor to the induction of EMT/EndoMT. The same finding can be observed in UVM. Oxidative stress causes dissociations of cell-cell adhesion of retinal pigment epithelial cells, which constitute the BRB, and TNF- $a$ induces the EMT of retinal pigmented epithelial cells $[19,20]$. The current study showed that inflammatory pathways such as the TNF-a, as well as EMT, were strongly activated in the high CTL group in UVM, indicating a possibility that inflammation had a significant influence on the induction of EMT/EndoMT. When BRB and BBB undergo EMT or EndoMT, their structures loosen and vascular permeability increases, thereby allowing the infiltration of large immune cells, such as macrophages, which normally cannot pass $[9,16]$. Gliomas are macrophage-dominant cancers, and they are correlated with an abundance of microglia, a type of glial cell, and resident macrophages, in the CNS. Previous studies have shown that in low-grade glioma, microglia are distributed closer to the center of tumor and circulating macrophages derived from monocytes invade the tumor beyond the BBB [21]. These findings suggest a positive feedback loop in which macrophages infiltrate a tumor and enhance inflammation, promoting EMT/EndoMT to the BRB and BBB, which in turn recruits additional inflammatory macrophages. In addition, EMT was not associated with a worse prognosis in GBM. In advanced gliomas, including GBM, the BBB is often disrupted due to tumor overgrowth. This is consistent with the hypothesis that EMT occurs in the cells comprising the BBB.

Furthermore, our results show M2 macrophages are abundant in UVM, while the proportion of M0 and M1 macrophages increased in GBM compared with LGG. This is consistent with the single-cell analysis data showing that inflammatory effects were observed in CD163 positive (M2-like) macrophages in UVM and CD163 negative (M0 or M1-like) macrophages in glioma. Hence, the inflammatory effect of M1-like macrophages may have a negative impact on prognosis in the CNS. In a mouse glioma model, the inflammatory effect of M1 macrophages under hypoxic conditions can damage even normal neuron cells [22]. Moreover, there are autoimmune diseases of the uvea that cause severe vision loss due to the inflammatory effects of resident immune cells [23]. Considering that the eye and CNS are immuneprivileged sites, where immune reactions are not likely to occur, these organs may be natively sensitive to inflammation.

Considering the possibility that the inflammatory effects from macrophages may induce EMT and EndoMT in BRB and BBB in UVM and LGG, loosening the structure and causing further macrophages infiltration, we suggest that repair of these structures and suppression of infiltration may be able to improve prognosis. To investigate this, we focused on chemokines. In recent years, check point inhibitors were developed to improve treatment modalities by regulating expression or inhibition of specific interleukins and chemokines [24]. Our results show that the expression of chemokine CCL 5 is strongly increased with immune activation and significantly associated with a worse prognosis. CCL5 inhibitors repair the disrupted BBB [24]. Moreover, microglias respond to CCL5 and promote the disruption of BBB in later stages via inflammatory effects [25]. These observations indicate that CCL5 has a potential to be a new molecular target. 


\section{Conclusion}

A high tumoral immune activity does not necessarily indicate a better prognosis, and the histological specificity of each organ may have a significant role. In UVM and LGG, which are cancers of immuneprivileged sites, the prognosis is better with low immune activity, even though this is contrary to the general concept of tumor immunity. The eye and CNS are protected by the blood barrier, which prevents the infiltration of immune cells. However, our result suggests that when immunity activated, EMT and EndoMT are induced in cells comprising the blood barrier, which is highly involved in bad prognosis in these cancers. Our data also shows promoted inflammation response and increased expression of CCL5 along with immune activation. These effects are known to cause further EMT/EndoMT and loosening the structure of the blood barrier. Therefore, we suggest that this mechanism may result in additional inflammatory effects through a deleterious positive feedback loop. Considering the histological impact of each cancer type, reducing excessive immune cell infiltration and maintaining the tissue structure could be a part of an effective immunotherapy strategy.

\section{Materials And Methods}

\section{Data preparation}

We used data available from The Cancer Genome Atlas (TCGA) project [26]. We downloaded the mRNA sequencing results and clinical information of 11057 samples from 33 different cancer types from the UCSC Xena (http://xenabroser.net/datapages/). The mRNA data were provided in FPKM, and the total number of reads for each sample was disparate. Therefore, the expression of each gene was transformed by multiplying by $1,000,000 /$ total number of reads. For validation, we used RNA-seq and clinical data of the Chinese Glioma Genome Atlas (CGGA), which is a public genomic database of Chinese patients with glioma $[27,28]$. The mRNA sequencing data and clinical information of 693 patients with glioma were downloaded. Gene expressions were provided as RSEM, and no specific correction was made.

We obtained single-cell RNA-seq data from eight primary UVM samples (GSE139829) [13] and nine glioma samples (GSE138794) [14]. The data were combined per cancer type using the Seurat package (4.0.0) in R (4.0.3). Filtering was conducted based on previous studies [13, 14]. For GSE139829 data, cells were retained that had unique molecular identifiers (UMIs) greater than 400, between 100 and 8000 expressed genes (inclusive), and less than 10\% mitochondrial content. For GSE138794 data, retained cells had UMls greater than 200, expressed more than 1 gene, and had mitochondrial content less than $5 \%$. Sample batch correction was not performed. Data were normalized using the LogNormalize method, and the scale factor was 10,000 . Visualization was conducted using Umap. As in previous studies [13, 14], we used markers for immune cells: CD14, CD68, and CD163 for macrophages; CLDN5 and VWF for endothelial cells; RPE65 for retinal pigment cells (GSE139829 only); MITF for cancer cells (GSE139829 only); and GFPA and AQP4 for astrocytes (GSE138794 only). 


\section{Immune scores, biological states and process activity in the tumor microenvironment}

The CTL level was used to indicate tumoral immune activity. The sum of the expression values of five genes ( $C D 8 A, C D 8 B, G Z M A, G Z M B$, and PRF1) was used to calculate the CTL level according to previous studies $[29,30]$.

We performed a single sample gene set enrichment analysis (SSGSEA) to determine the activity level of each signaling pathway, states, or processes in the gene expression profile. The GSVA package (1.34.0) in $\mathrm{R}$ (3.6.3) was used for bulk RNA-seq data analysis with the following parameters: method = ssgsea, tau = 0.75 , ssgsea.norm $=F$. Data were zero-mean normalized [31]. The script was implemented with reference to the code of Immunoduct (https://github.com/msfuji/immunoduct/tree/master/scripts). For single-cell RNA-seq samples, the escape package (1.1.1) in R (4.0.3) was utilized to perform ssGSEA [32]. Moreover, the Hallmark gene sets, which are registered in the Molecular Signatures Database (MSigDB) version 7.4, were applied [33].

We ran cell-type identification by estimating the relative subsets of RNA transcripts (CIBERSORT) to estimate the abundances of various immune cells in the TME based on the mRNA expression profile [34]. We used LM22 as the signature gene file, and permutation was set to 100 . The command line script for CIBERSORT version 1.04 was used in $\mathrm{R}$ (3.6.3), and only results with a $p$-value of $<0.05$ were utilized. When determining the percentage of each immune cell in the tumor, absolute mode was set to false. The script was implemented with reference to the code of Immunoduct. In addition, we used another signature-based method to infer immune cells (xCell). xCell data from the TCGA are available on the website [35], and they were used to validate CIBERSORT results.

\section{Statistical analysis}

Survival and statistical analyses were conducted using the lifelines package (0.25.9) and the scipy package (1.6.1) in Python (3.8.5). Kaplan-Meier curves were used to assess the influence of different factors on patient survival, and the log-rank test was utilized to calculate the $p$-value. The patients were divided into two groups using the median of each calculated score, and hazard ratios for patient prognosis were calculated utilizing the Cox proportional hazard regression model, with samples below the median as 0 and those above the median as 1 . Data between the two groups were compared using the Welch's $t$-test, and the Pearson's correlation coefficient was used to evaluate correlations. A $p$-value of < 0.05 was considered statistically significant. Bonferroni's correction was applied for multiple testing.

\section{Abbreviations}

BBB, blood-brain barrier; BRB, blood-retinal barrier; CNS, central nervous system; CTL, cytotoxic T lymphocyte; EndoMT, endothelial mesenchymal transition; EMT, epithelial mesenchymal transition; GBM, glioblastoma; HR, hazard ratio; LGG, low-grade glioma; SKCM, skin cutaneous melanoma; TME, tumor microenvironment; UVM, uveal melanoma 


\section{Declarations}

\section{Ethics approval and consent to participate}

Not applicable

\section{Consent for publication}

Not applicable

\section{Availability of data and materials}

All data used in this study are included in these published articles $[13,14,26,27,28]$.

\section{Competing interests}

The authors declare no competing interests.

\section{Funding}

This work was funded by JST CREST Grant Number JPMJCR 1412, Japan; JSPS KAKENHI Grant Numbers JP17H06307, JP17H06299, JP20H03240, and JP20K16408; Grant-in-Aid for Scientific Research (JP16H06299) from the Ministry of Education, Culture, Sports, Science and Technology of Japan.

\section{Author contributions}

$\mathrm{HM}, \mathrm{TK}$ and TT conceived the research idea. HM performed data analysis. HM, TK, YH and TT interpreted the result. HM, TK, KB and TT contributed to the writing and revision of the manuscript. All authors approved the final article and agreed to be accountable for all aspects of the work.

\section{Acknowledgements}

We are grateful to Dr. Yoshihiro Kushihara and Professor Kazuhiro Kakimi, Department of Immunotherapeutics, The university of Tokyo Hospital, for fruitful discussion on this research. The authors would like to thank Enago (www.enago.jp) for the English language review.

\section{References}

1. Camus M, Tosolini M, Mlecnik B, et al. Coordination of intratumoral immune reaction and human colorectal cancer recurrence. Cancer Res. 2009;69:2685-2693.

2. Galon J, Bruni D. Approaches to treat immune hot, altered and cold tumours with combination immunotherapies. Nat Rev Drug Discov. 2019;18:197-218. 
3. Haanen JB. Converting cold into hot tumors by combining immunotherapies. Cell. 2017;170:10551056.

4. Ribas A, Dummer R, Puzanov I, et al. Oncolytic virotherapy promotes intratumoral T cell infiltration and improves anti-PD-1 immunotherapy. Cell. 2017;170:1109-1119.

5. Zemek RM, De Jong E, Chin WL, et al. Small M. Sensitization to immune checkpoint blockade through activation of a STAT1/NK axis in the tumor microenvironment. Sci Transl Med. 2019;11:eaav7816.

6. Duan Q, Zhang H, Zheng J, Zhang L. Turning cold into hot: firing up the tumor microenvironment. Trends Cancer. 2020;6:605-618

7. Danaher $P$, Warren $S$, Lu R, et al. Pan-cancer adaptive immune resistance as defined by the Tumor Inflammation Signature (TIS): results from The Cancer Genome Atlas (TCGA). J Immunother Cancer. 2018;6:1-7.

8. Yang $X$, Shi Y, Li M, et al. Identification and validation of an immune cell infiltrating score predicting survival in patients with lung adenocarcinoma. J Transl Med. 2019;17:1-9.

9. Troletti CD, de Goede P, Kamermans A, de Vries HE. Molecular alterations of the blood-brain barrier under inflammatory conditions: the role of endothelial to mesenchymal transition. Biochim Biophys Acta Mol Basis Dis. 2016;1862:452-460.

10. Gonzalez DM, Medici D. Signaling mechanisms of the epithelial-mesenchymal transition. Sci Signal. 2014;7:re8.

11. Iwadate Y. Epithelial-mesenchymal transition in glioblastoma progression. Oncol Lett. 2016;11:16151620.

12. Shu DY, Butcher E, Saint-Geniez M. EMT and EndMT: emerging roles in age-related macular degeneration. Int J Mol Sci. 2020;21:4271.

13. Durante MA, Rodriguez DA, Kurtenbach S, et al. Livingstone AS, Harbour JW. Single-cell analysis reveals new evolutionary complexity in uveal melanoma. Nat Commun. 2020;11:1-10.

14. Wang L, Babikir H, Müller S, et al. The phenotypes of proliferating glioblastoma cells reside on a single axis of variation. Cancer Discov. 2019;9:1708-1719.

15. Rudraraju M, Narayanan SP, Somanath PR. Regulation of blood-retinal barrier cell-junctions in diabetic retinopathy. Pharmacol Res. 2020:105115.

16. Crane IJ, Liversidge J. Mechanisms of leukocyte migration across the blood-retina barrier. Semin Immunopathol 2008;30:165-177.

17. Engelhardt B, Vajkoczy P, Weller RO. The movers and shapers in immune privilege of the CNS. Nature Immunol. 2017;18:123.

18. Chuntova P, Downey KM, Hegde B, Almeida ND, Okada H. Genetically engineered T-cells for malignant glioma: overcoming the barriers to effective immunotherapy. Front Immunol. 2019;9:3062.

19. Inumaru J, Nagano O, Takahashi E, et al. Molecular mechanisms regulating dissociation of cell-cell junction of epithelial cells by oxidative stress. Genes Cells 2009;14:703-716. 
20. Takahashi E, Nagano $O$, Ishimoto $T$, et al. Tumor necrosis factor-a regulates transforming growth factor- $\beta$-dependent epithelial-mesenchymal transition by promoting hyaluronan-CD44-moesin interaction. J Biol Chem. 2010;285:4060-4073.

21. Müller S, Kohanbash G, Liu SJ, et al. Single-cell profiling of human gliomas reveals macrophage ontogeny as a basis for regional differences in macrophage activation in the tumor microenvironment. Genome Biol. 2017;18:1-4.

22. Hu X, Leak RK, Shi Y, et al. Microglial and macrophage polarization-new prospects for brain repair. Nat Rev Neurol. 2015;11:56.

23. Okunuki Y, Mukai R, Nakao T, et al. Retinal microglia initiate neuroinflammation in ocular autoimmunity. Proc Natl Acad Sci. 2019;116:9989-9998.

24. Saha D, Martuza RL, Rabkin SD. Macrophage polarization contributes to glioblastoma eradication by combination immunovirotherapy and immune checkpoint blockade. Cancer Cell. 2017;32:253-267.

25. Seo W, Shimizu K, Kojo S, et al. Runx-mediated regulation of CCL5 via antagonizing two enhancers influences immune cell function and anti-tumor immunity. Nat Commun. 2020;11:1-6.

26. Weinstein JN, Collisson EA, Mills GB, Shaw KR, Ozenberger BA, Ellrott K, Shmulevich I, Sander C, Stuart JM. The cancer genome atlas pan-cancer analysis project. Nat Gen. 2013;45:1113-1120.

27. Liu X, Li Y, Qian Z, et al. A radiomic signature as a non-invasive predictor of progression-free survival in patients with lower-grade gliomas. Neurolmage Clin. 2018;20:1070-1077.

28. Wang Y, Qian T, You G, et al. Localizing seizure-susceptible brain regions associated with low-grade gliomas using voxel-based lesion-symptom mapping. Neuro-Oncol. 2015;17:282-288.

29. Jiang P, Gu S, Pan D, et al. Signatures of T cell dysfunction and exclusion predict cancer immunotherapy response. Nat Med. 2018;24:1550-1558.

30. Rooney MS, Shukla SA, Wu CJ, Getz G, Hacohen N. Molecular and genetic properties of tumors associated with local immune cytolytic activity. Cell. 2015;160:48-61.

31. Hänzelmann S, Castelo R, Guinney J. GSVA: gene set variation analysis for microarray and RNA-seq data. BMC Bioinform. 2013;14:1-5.

32. Borcherding N, Vishwakarma A, Voigt AP, Bellizzi A, Kaplan J, Nepple K, Salem AK, Jenkins RW, Zakharia $Y$, Zhang W. Mapping the immune environment in clear cell renal carcinoma by single-cell genomics. Commun Biol. 2021;4:1-11.

33. Liberzon A, Birger C, Thorvaldsdóttir H, Ghandi M, Mesirov JP, Tamayo P. The molecular signatures database hallmark gene set collection. Cell Syst. 2015;1:417-425.

34. Newman AM, Liu CL, Green MR, et al. Robust enumeration of cell subsets from tissue expression profiles. Nat Methods. 2015;12:453-457.

35. Aran D, Hu Z, Butte AJ. xCell: digitally portraying the tissue cellular heterogeneity landscape. Genome Biol. 2017;18:1-4.

\section{Figures}


a
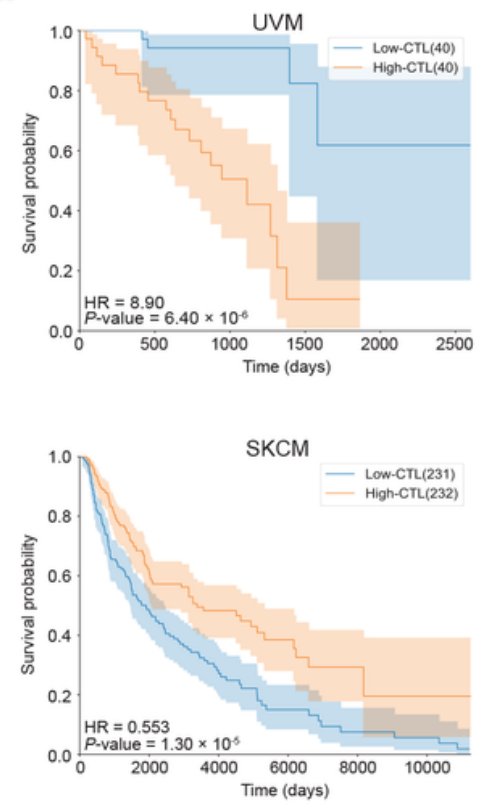
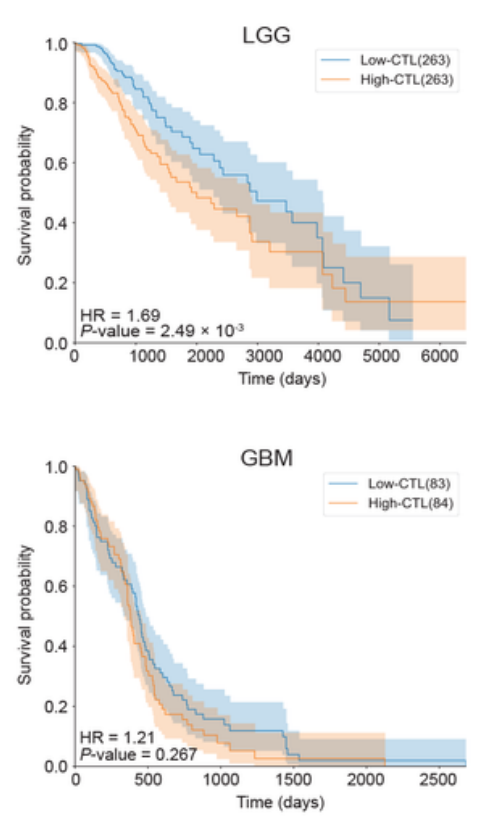

b

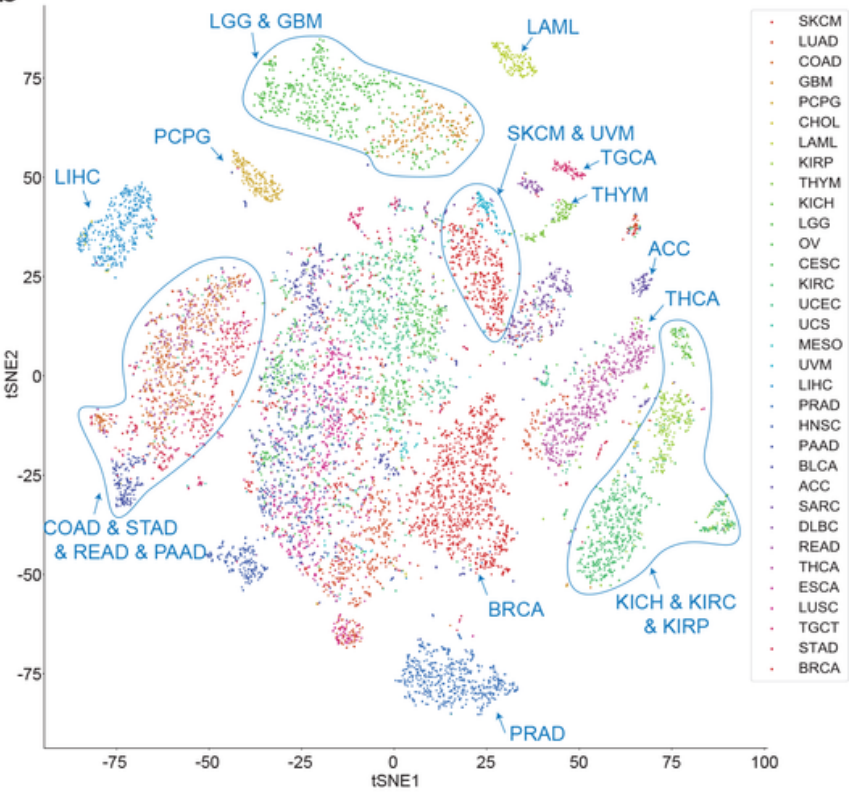

Figure 1

High immune activity was associated with poor prognosis in patients with UVM and LGG. a Kaplan-Meier curves of overall survival classified according to CTL levels (red: high CTL level, blue: low CTL level). The number samples are indicated in the legend. $b$ tSNE plot based on the activity of well-defined biological states and processes calculated for all samples and color-coded according to cancer type. The names of the cancer types follow TCGA Study Abbreviations. EMT, epithelial mesenchymal transition.
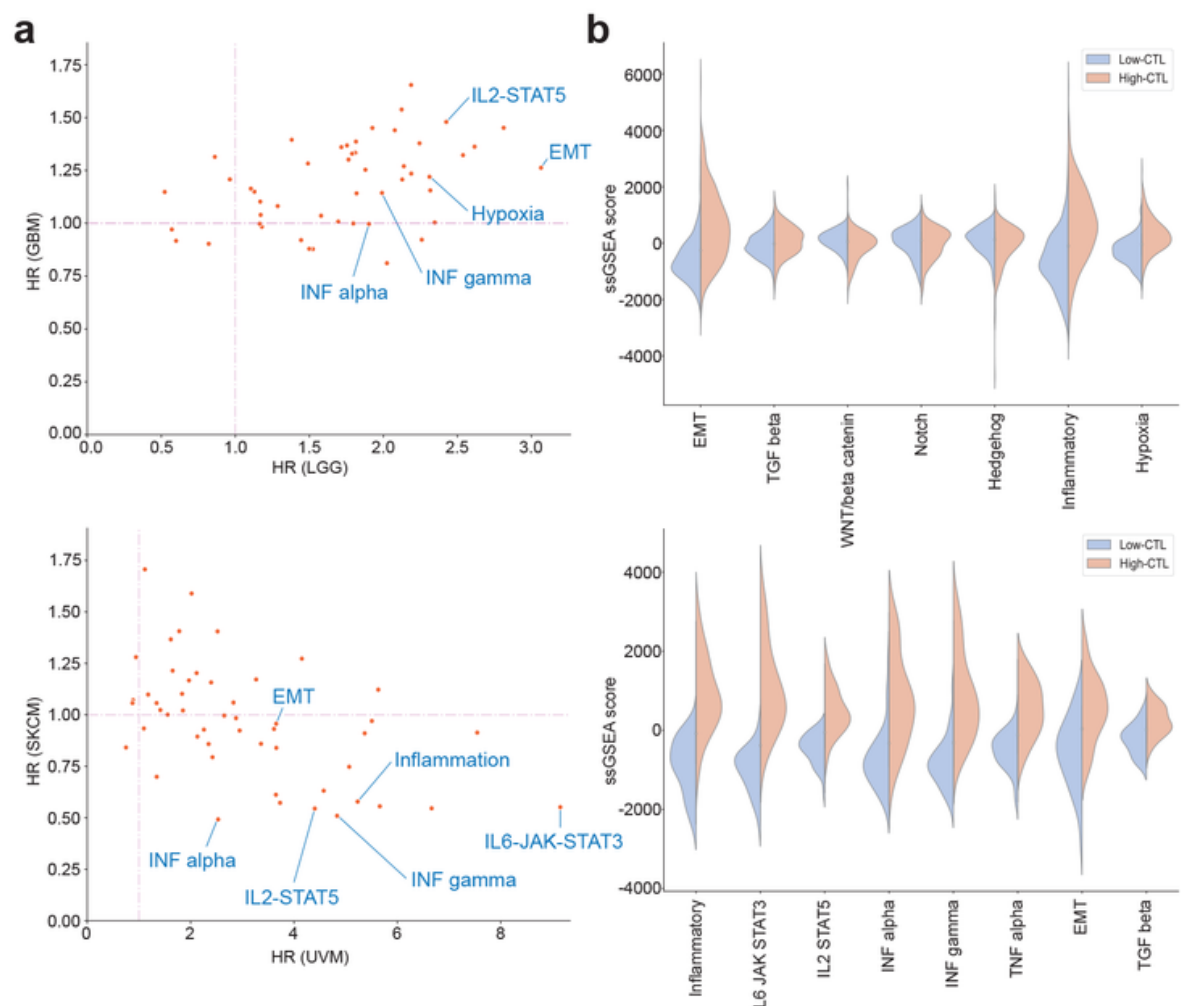
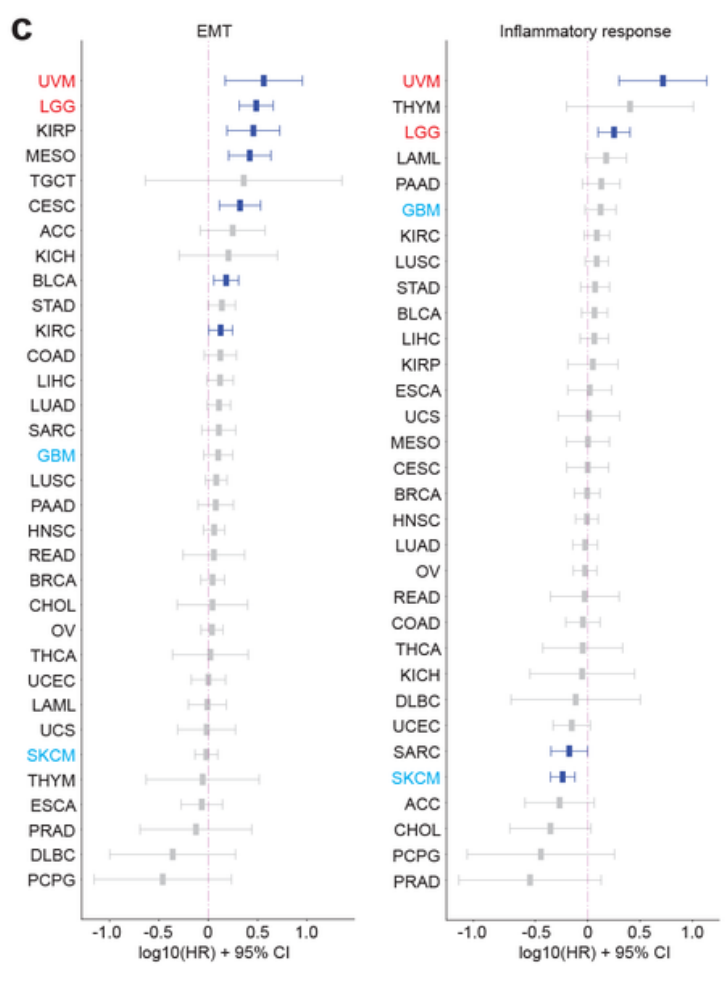

Figure 2 
Hazard ratios of inflammation and EMT in UVM and LGG. a Hazard ratio of each state and process in LGG and GBM (top) and in UVM and SKCM (bottom). b Difference in activity score for some states and processes between the high and low CTL groups in LGG (top) and UVM (bottom). c Logarithm of hazard ratios for EMT (left) and inflammatory response (right) among different cancer types. Those with a pvalue of $<0.05$ are depicted in blue and others in gray. The names of the cancer types follow TCGA Study Abbreviations. EMT, epithelial mesenchymal transition.

a

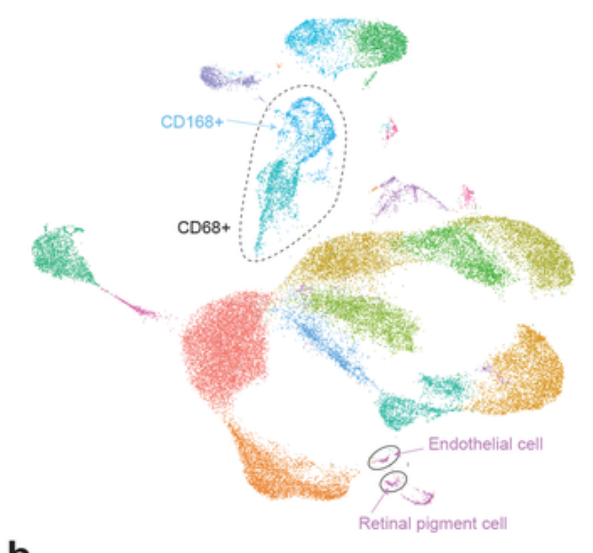

b

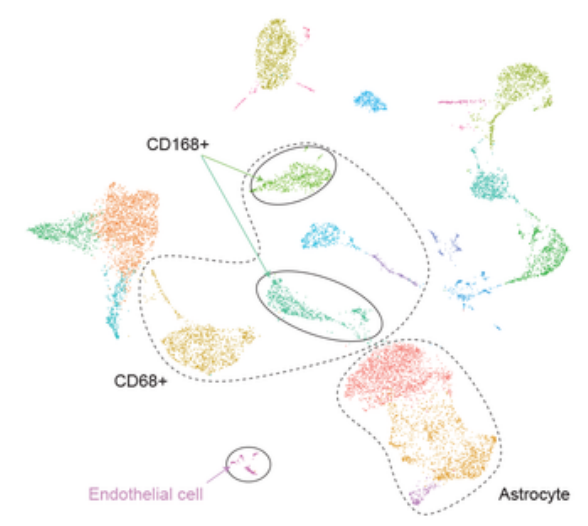

C
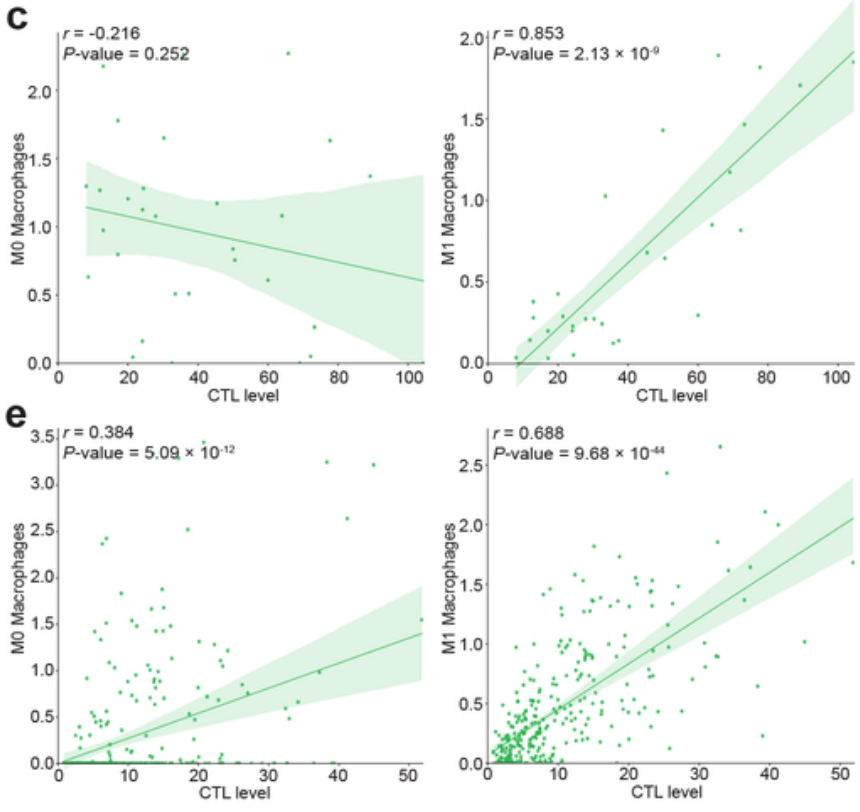

Inflammatory response

Inflammatory response
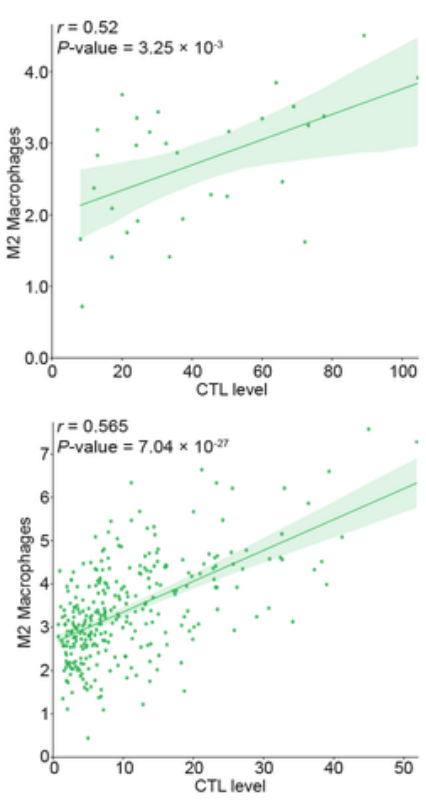

EMT

EMT

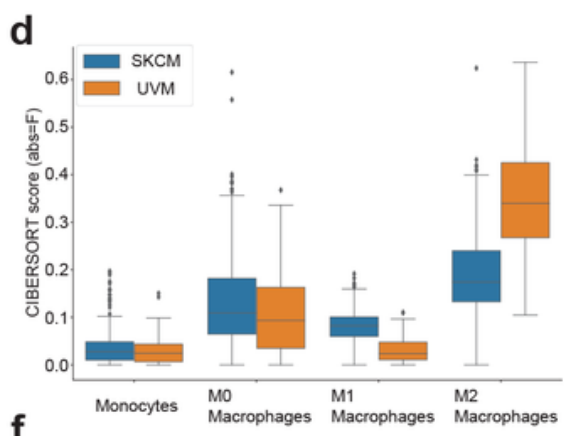

Figure 3 
Single-cell analysis and the macrophage infiltration level in UVM and LGG. a, b Umap projection of clustering analysis (left), and the overview of activation scores of inflammation (middle) and EMT (right) across all cells in UVM (a) and LGG (b). c, d Correlation between M0, M1, and M2 macrophage levels determined using CIBERSORT (absolute=T) and CTL levels in UVM (c) and LGG (d). e, $f$ The fraction of monocyte and $\mathrm{MO}, \mathrm{M} 1$, and $\mathrm{M} 2$ macrophages in the tumor determined using CIBERSORT (absolute=F) in UVM and SKCM (e) and LGG and GBM (f). SKCM, skin cutaneous melanoma; UVM, uveal melanoma; LGG, low-grade glioma; GBM, glioblastoma.
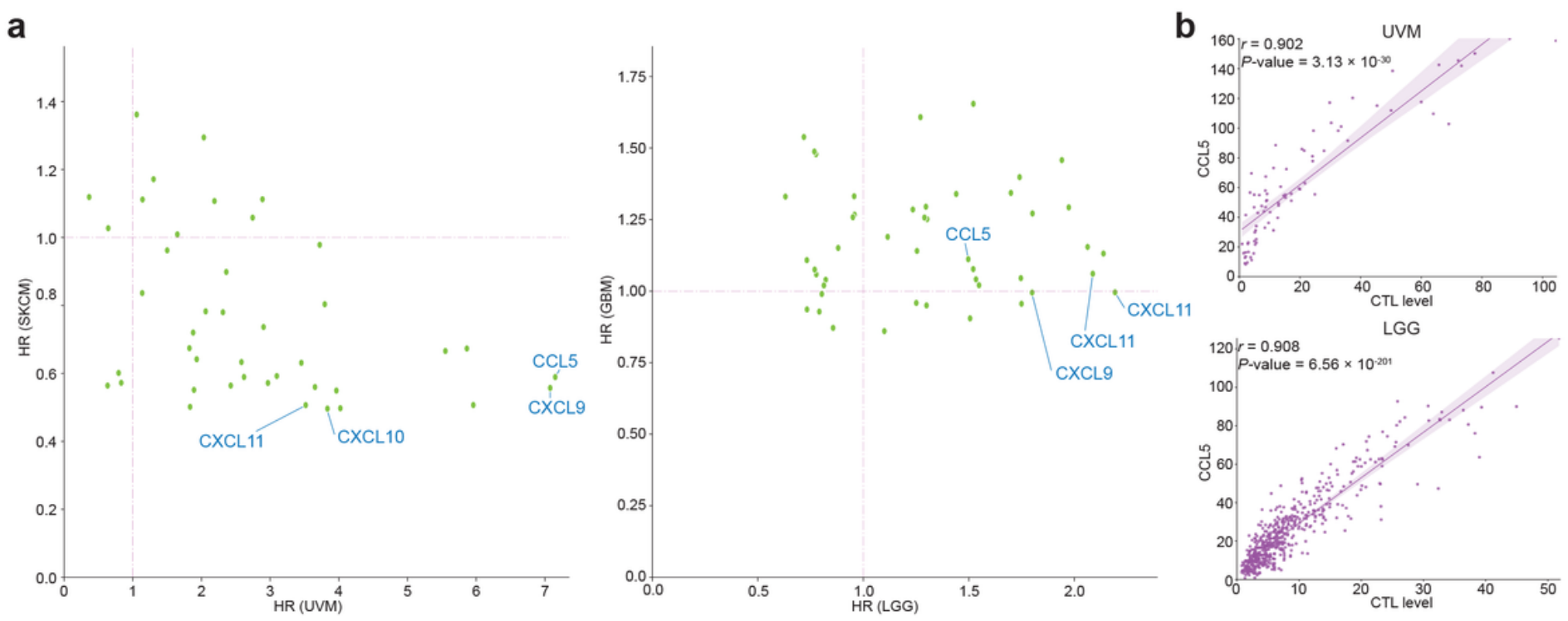

\section{Figure 4}

Association between chemokine expression as well as overall survival and CTL levels. a Hazard ratio of each chemokine in UVM and SKCM (left) and LGG and GBM (right). b Correlation between CCL5 expression and CTL levels in UVM (top) and LGG (bottom). SKCM, skin cutaneous melanoma; UVM, uveal melanoma; LGG, low-grade glioma; GBM, glioblastoma.

\section{Supplementary Files}

This is a list of supplementary files associated with this preprint. Click to download.

- Supplementaryfigures.pdf

- Supplementarytable.docx 